\title{
Selection of Surgical Approach for Cerebellar Hemangioblastomas Based on Venous Drainage Patterns
}

\section{Takashi Watanabe ( $\sim$ takashi_watanabe@med.miyazaki-u.ac.jp )}

University of Miyazaki Faculty of medicine https://orcid.org/0000-0002-3724-1962

\section{Yuuki Suematsu}

Department of Neurosurgery, Faculty of Medicine, University of Miyazaki, Miyazaki, Japan

\section{Kiyotaka Saitou}

Department of Neurosurgery, Faculty of Medicine, University of Miyazaki, Miyazaki, Japan

\section{Go Takeishi}

Department of Neurosurgery, Faculty of Medicine, University of Miyazaki, Miyazaki, Japan

\section{Shinji Yamashita}

Department of Neurosurgery, Faculty of Medicine, University of Miyazaki, Miyazaki, Japan

\section{Hajime Ohta}

Department of Neurosurgery, Faculty of Medicine, University of Miyazaki, Miyazaki, Japan

\section{Kiyotaka Yokogami}

Department of Neurosurgery, Faculty of Medicine, University of Miyazaki, Miyazaki, Japan

Hideo Takeshima

Department of Neurosurgery, Faculty of Medicine, University of Miyazaki, Miyazaki, Japan

\section{Research Article}

Keywords: cerebellum, cerebral veins, hemangioblastoma, surgical approach

Posted Date: February 15th, 2021

DOI: https://doi.org/10.21203/rs.3.rs-229544/v1

License: (1) (1) This work is licensed under a Creative Commons Attribution 4.0 International License. Read Full License

Version of Record: A version of this preprint was published at Neurosurgical Review on April 20th, 2021. See the published version at https://doi.org/10.1007/s10143-021-01544-y. 


\section{Abstract}

Cerebellar hemangioblastomas remain surgically challenging because of the narrow, deep surgical corridors and tumor hypervascularity. Various surgical approaches are used according to the location, but optimal approaches have not been established. We propose a system of surgical approaches based on the venous drainage systems to facilitate surgical planning and achieve acceptable neurological outcomes. Cerebellar hemangioblastomas were divided into fivefour types based on the main drainage systems: suboccipital hemangioblastomas draining to the transverse sinus (TS)_or torcular, tentorial hemangioblastomas draining to the tentorial and straight sinus, petrosal hemangioblastomas draining to the superior petrosal sinus (SPS),, and quadrigeminal hemangioblastomas draining to the galenic system, and tonsillar hemangioblastomas draining to the TS or torcular in conjunction with jugular bulb or SPS. Microsurgical approaches and patient outcome were retrospectively reviewed according to this classification. This study included 17 patients who underwent 21 operations for resection of 19 cerebellar hemangioblastomas, classified into $\underline{9} 11$ suboccipital, 4 tentorial, 2 petrosal, and 2 quadrigeminal, and 2 tonsillar. Standard suboccipital craniotomies were utilized for suboccipital hemangioblastomas, the occipital transtentorial approach (OTA) and supracerebellar infratentorial approach for tentorial hemangioblastomas, the retrosigmoid approach for petrosal hemangioblastomas, and OTA for quadrigeminal hemangioblastomas, and midline suboccipital approach for tonsillar hemangioblastomashemangioblastomass. Gross total resection was achieved in all patients except one. Two patients with large hemangioblastomas (tonsillarsuboccipital and quadrigeminal) required secondstage operation which finally achieved gross total removal. No single approach had a significantly higher incidence of postoperative neurological deficits. Selection of the optimum surgical approach for cerebellar hemangioblastomas was successful based on the main drainage systems. Understanding of tumor growth and extension with respect to the venous drainage system is critical to select the appropriate surgical approach.

\section{Introduction}

Central nervous system (CNS) hemangioblastoma is a relatively rare intracranial neoplasm accounting for $1.5-2.5 \%$ of CNS tumors, and can occur as a sporadic entity as well as in patients with von HippelLindau (VHL) disease, an autosomal dominant inherited disorder.[10,14] CNS hemangioblastomas are benign, highly vascularized tumors classified as grade I according to the World Health Organization classification. Despite the benign histological features of the tumor, mass effect resulting from tumor progression, peritumoral cyst formation, and expanding peritumoral edema may cause morbidity and mortality.[34] Surgery is curative and the treatment of choice in most patients with clinical symptoms. The goal of surgery is complete removal of the lesion to prevent perioperative hemorrhage and to preserve surrounding neurovascular functions.

CNS hemangioblastomas occur most frequently in the cerebellum followed by other specific CNS locations such as the retina, spinal cord, and brainstem.[6,13,34] Various surgical approaches for cerebellar hemangioblastoma are adopted according to tumor location. [1,7-11,15,20,21,33] Cerebellar 
hemangioblastomas present unique operative challenges, despite current advances in microneurosurgical techniques, such as the narrow and deep surgical corridor in the posterior fossa, inability to debulk the tumor mass due to hypervascularity, difficulty in early access to the feeding artery behind the tumor, and interference by large tortuous draining veins during the resection. [8-10,19,21] Consequently, selection of the optimal surgical approach and operative planning is quite important to access the pial surface and achieve panoramic exposure of the circumferential entire margin of the lesion for early management of the cortical feeding artery and identification of the main draining system. However, the optimal surgical approach to cerebellar hemangioblastomas has not been systematically established and comprehensively specified because of the rarity of these lesions.

The current study tried to systematize our surgical approach and evaluated our surgical results for 19 cerebellar hemangioblastomas according to a classification of the cerebellar surface into five different regions based on the venous drainage system. We found that these subtypes offer intuitive appreciation of their anatomy and surgical management and are useful in describing the surgical results.

\section{Materials \& Methods}

\section{Patient Population}

Nineteen consecutive patients underwent a total of 24 operations for resection of 22 cerebellar hemangioblastomas at the University of Miyazaki between January 2007 and March 2020. Three recurrent hemangioblastomas in 2 patients were excluded because the drainage veins could not be determined due to the previous surgery, so finally 17 patients who underwent a total of 21 operations for 19 cerebellar hemangioblastomas were evaluated. Medullary and spinal hemangioblastomas were not included in this study. The diagnosis of VHL disease was made according to the clinical criteria. Clinical data pertaining to patient demographics, neurological status, operative record, and clinical outcome were obtained by retrospective chart review. All available baseline medical data were assessed, including medical records, neuroimaging studies, intraoperative photographs, and videos.

All patients underwent 3.0 T magnetic resonance (MR) imaging (contrast-enhanced T1-weighted and fluid-attenuated inversion recovery [FLAIR] images), and three-dimensional computed tomography angiography to evaluate the tumor location, maximum tumor diameter, course of the feeding artery, and draining veins. Maximum tumor and/or cyst diameter were measured and classified into small ( $<25 \mathrm{~mm})$ and large ( $\geq 25 \mathrm{~mm}$ ) tumors. Intraoperative findings were systematically reviewed on the operative records and videos. Extent of resection was determined by retrospective review of the postoperative MR images obtained within 3 days after surgery. Histological diagnosis was made according to the criteria of the World Health Organization classification.[4,14] Detailed neurological assessments were performed by neurosurgical residents and the senior neurosurgeon, preoperatively and postoperatively. Neurological outcome was assessed with the modified Rankin Scale (mRS). Good and pooroutcomes were definedas final $m R S \leq 2$ and $m R S>2$. Neurological improvement and deterioration were defined as decrease and 
increase in mRS score, respectively. Sixteen hemangioblastomas with 14 patients were followed up for at least 5 months with serial clinical and MR imaging examinations at 6- or 12-month intervals.

\section{Classification of Cerebellar Hemangioblastomas}

Cerebellar hemangioblastomas were organized into the following five groups according to their drainage venous system (Fig. 1).

Suboccipital Hemangioblastoma

Suboccipital hemangioblastoma is located on the suboccipital and tonsillar surface and drains toward the inferior vermian veins, inferior hemispheric veins, or both, which empty into the transverse sinus or torcular.

Tentorial Hemangioblastoma

Tentorial hemangioblastoma faces the tentorial surface and is located in the extreme narrow space along the supracerebellar-infratentorial plane. Venous drainage is through the superior hemispheric veins, which empty into the straight sinus or tentorial sinus.

Petrosal Hemangioblastoma

Petrosal hemangioblastoma faces the corner of the petrosal surface and tentorium, and drained by the petrosal veins, which empty into the superior petrosal sinus (SPS).

Quadrigeminal Hemangioblastoma

Quadrigeminal hemangioblastoma is located on the anteromedial cerebellar surface facing the quadrigeminal cistern, and drained by the precentral cerebellar vein or superior vermian vein, which belong to the galenic system that drains into the vein of Galen.

Tonsillar Hemangioblastoma

Tonsillar hemangioblastoma is located on the tonsillar surface and drains toward the superficial venous system which empties into the transverse sinus or torcular, in conjunction with the deep venous drainage system emptying into the jug gular bulb or SPS.

\section{Microsurgical Technique}

The same fundamental surgical technique is used for resection of all hemangioblastomas after craniotomy with the selected surgical approach for different tumor locations. Dural incision is made to gain access to the exposed tumor-pial border, surrounding feeding arteries, and draining veins. The tumorpial interface is sharply dissected and feeding arteries are sharply interrupted, followed by deep circumferential dissection at the tumor-cerebellar tissue interface. The draining veins and major draining venous systems, which are often prominent in the surgical field and obstruct the resection, should be 
meticulously dissected and preserved until the end of the surgery. Tumor shrinkage by step-wise bipolar coagulation of the tumor capsule provides better visualization of the deep tumor-cerebellar interface. Care should be taken to preserve venous drainers of some hemangioblastomas during dissection of the deep tumor-cerebellar interface. Finally, en-bloc resection can be achieved after coagulation and ligation of the draining veins. For lesions associated with peritumoral cysts, the entire cyst wall is left and carefully inspected after tumor removal. External ventricular drainage is placed to manage hydrocephalus and reduce raised intracranial pressure.

\section{Preoperative Embolization}

Standard transfemoral angiographic technique was used to obtain global angiographic images in 15 patients to evaluate the vascular structures. Preoperative liquid embolization procedures were performed under local anesthesia for 8 hemangioblastomas within 24 hours before surgery. After careful evaluation of the superselective angiography using flow-directed microcatheters of the safely accessible, most peripheral feeding arteries supplying the tumor, embolization of strictly selected arterial feeders was performed using N-butyl-2-cyanoacrylate (NBCA) adjusted to adequate concentrations to avoid occlusion of the drainers.

\section{Results}

\section{Patient Demographics}

This study included 17 patients who underwent a total of 21 operations for resection of 19 cerebellar hemangioblastomas at our institution, 10 men and 7 women aged 23-86 years (mean age $53.6 \pm 20.8$ years) at initial surgery. Eight patients had sporadic etiology and 9 met the clinical criteria for the diagnosis of VHL disease. Demographic and baseline clinical data were available for all patients (Table 1). Most patients (10 of 17) presented with cerebellar ataxia. Five patients presented with headache. One patient presented with facial numbness. One patient had progressive disturbance of consciousness due to acute hydrocephalus. The clinical characteristics of 19 hemangioblastomas are shown in Table 2 . Mean maximum diameter was $33.7 \pm 12.9 \mathrm{~mm}$. Fifteen hemangioblastomas $(78.9 \%)$ were classified as large. Eleven patients had solitary and 8 had multiple lesions. Peritumoral cysts and intratumoral cysts were associated with 8 and 2 hemangioblastomas, respectively. The other 9 tumors had solid morphology. Thirteen hemangioblastomas (68.4\%) were associated with obstructive hydrocephalus. Eighteen of the 19 hemangioblastomas (94.7\%) were associated with peritumoral edema. Extensive and local peritumoral edema was demonstrated on FLAIR sequences in 12 and 6 hemangioblastomas, respectively. One suboccipital hemangioblastoma with large peritumoral cyst was not associated with peritumoral edema.

\section{Classification of Cerebellar Hemangioblastomas and Clinical Outcomes}

Characteristics and surgical approaches selected for the different subtypes are shown in Table 3. 
This study included 9 (47.4\%) suboccipital hemangioblastomas, which drained to the torcula and transverse sinuses from the inferior vermian veins (3 of $11,27.3 \%$ ), inferior hemispheric veins ( $\underline{5}$ of 11 , $63.6 \%$ ), or both (1 of $11,9.1 \%$ ). Suboccipital hemangioblastomas are the most common and located on the suboccipital surface (Fig. 2), which is the most accessible and so could be removed through standard suboccipital craniotomies according to the tumor size. These craniotomies provide panoramic exposure of the entire margin of the lesions, which allows early identification and control of the feeding arteries supplied by cortical branches from the superior cerebellar artery (SCA) from superiorly, posterior inferior cerebellar artery (PICA) from inferiorly, and anterior inferior cerebellar artery (AICA) from the lateral margin. These craniotomies also allow meticulous dissection under direct visualization and preservation of the draining veins until the end stage of resection. Gross total removal was achieved in 9 of 10 patients. Small unexpected residual tumor was observed on postoperative MR imaging of one suboccipital hemangioblastoma with large intratumoral cyst. No permanent neurological complications occurred. All patients with suboccipital hemangioblastomas improved and remained stable.

Tentorial Hemangioblastomas

This study included 4 tentorial hemangioblastomas (21.1\%), which originated on the tentorial surface and extended along the extremely narrow space of the supracerebellar-infratentorial plane (Fig. 3). Venous drainage passed through the superior hemispheric veins, which directly drained to the straight sinus in all tumors. Tentorial hemangioblastomas were supplied by SCA feeders from the anterior margin of the lesion and PICA feeders from the posterior and AICA feeders from the anterolateral margins. In this series, three tentorial hemangioblastomas (75\%) located in the midline were resected through the occipital transtentorial approach (OTA) to gain access to the entire tumor-pial margin along the superior cerebellar surface. This approach requires careful consideration during tentorial incision to avoid potentially devastating hemorrhage from the tentorial sinus and to protect the main drainers. One tentorial hemangioblastoma with lateral extension was resected using the supracerebellar infratentorial approach (SCITA) for direct access to the posterior tumor surface. The suboccipital craniotomy was extended superiorly above the transverse sinus to elevate the tentorium and inferiorly to the foramen magnum. In this approach, preoperative embolization of the SCA feeders was beneficial because of the deep perforator supply from the SCA behind the tumor. Gross total removal was achieved in all four patients. Transient homonymous hemianopsia was noted in the 3 patients after the OTA, but no permanent neurological deficits occurred. All patients with tentorial hemangioblastomas improved after surgery.

Petrosal Hemangioblastomas

This study included 2 petrosal hemangioblastomas (10.5\%), located in the lateral portion of the cerebellum facing the corner between the petrous bone and tentorium (Fig. 4). The main feeder to these lesions was SCA dominant and collaborated with the AICA and PICA. Venous drainage mainly coursed to the petrosal sinus through the petrosal veins and superior hemispheric veins. Petrosal 
hemangioblastomas were resected through retrosigmoid craniotomy with exposure of the transversesigmoid junction to gain access to the petrosal surface and SPS. This approach can access a wide space for early evacuation of hematoma and relaxation of swollen cerebellum in patients with postembolic hemorrhage. The lesion was then dissected circumferentially and totally removed. Gross total removal was achieved in both patients, but new neurological deficits developed and mRS also worsened in one patient with postembolic hemorrhage.

Quadrigeminal Hemangioblastomas

This study included 2 quadrigeminal hemangioblastomas (10.5\%), which drained through the galenic system via the precentral cerebellar veins or superior vermian veins (Fig. 5). Feeding arteries were mainly supplied by cortical branches from the SCA supplemented by the PICA inferiorly and AICA laterally. Quadrigeminal hemangioblastomas are most challenging because of the deep location in the superior vermis and anteromedial region of the superior cerebellum facing the quadrigeminal plate, but can be accessed through the OTA. This approach provides good exposure of the tumor-pial interface and allows meticulous dissection of this interface and early control of the feeding arteries under direct visualization of the galenic system. Complete tumor resection was achieved in both lesions and confirmed on postoperative MR imaging. One quadrigeminal hemangioblastoma larger than $35 \mathrm{~mm}$ underwent secondstage surgery to complete resection through the OTA on both sides (Fig 5). Permanent unilateral homonymous hemianopsia was observed in the latter patient. Improvement or stabilization of $\mathrm{mRS}$ was observed in both patients.

Tonsillar hemangioblastoma

This study included 2 tonsillar hemangioblastomas (10.5\%), which originated on the tonsil with superficial drainage through the torcular or transverse sinus (Fig. 6). Deep drainage into the jugular bulb or SPS was also found. Feeding arteries were mainly supplied by cortical branches from the PICA supplemented by the AICA laterally and SCA superiorly. Tonsillar hemangioblastoma was classified into a different category from suboccipital hemangioblastoma because the deeper location with deep drainage makes the surgical procedure more challenging. The entire posterior surface was hidden by the cerebellar hemisphere and the anterior surface was adjacent to the medulla oblongata and lateral recess of the fourth ventricle. Tonsillar hemangioblastoma can be resected through midline suboccipital craniotomy including the posterior edge of the foramen magnum with or without $\mathrm{C} 1$ laminectomy. This approach can maintain the dissection plane of the tumor-cerebellar interface under direct visualization of the brainstem, main trunk of PICA, and superficial drainer, but careful attention should be given to any deep drainage vessel on the bottom of the lesion. One tonsillar hemangioblastoma larger than $40 \mathrm{~mm}$ required secondstage surgery for complete resection (Fig. 6).

\section{Surgical Results and Outcomes}

Complete resection was achieved in 18 of 19 hemangioblastomas (94.7\%) using our proposed surgical approaches (Table 3 ). None of the draining veins were sacrificed until the final stage of resection. 
Intraoperative placement of ventricular drains for cerebrospinal fluid diversion was required in 14 of 21 surgeries. Preoperative hydrocephalus resolved without ventriculoperitoneal shunt placement in all patients after surgical resection. No major complications were related to the procedures. No single approach had a significantly higher incidence of postoperative neurological deficits. One patient with VHL disease underwent 2 operations for 3 different lesions. Two patients with large solid hemangioblastomas (tonsillar and quadrigeminal) underwent two-staged surgery through the same surgical approach 2 weeks apart. Transient homonymous hemianopsia was observed in 4 of 6 patients after surgery using the OTA, but these symptoms improved within a few weeks. One patient with quadrigeminal hemangioblastoma experienced transient trochlear nerve palsy, which was resolved within several weeks. One patient with large quadrigeminal hemangioblastoma had persistent unilateral homonymous hemianopsia. Two patients who presented without clinical signs and symptoms before surgery remained asymptomatic. Three patients with preoperative mRS score of 4 improved to score of 3 in 2 patients and score of 4 remained in one patient. Finally, 13 of 17 patients had excellent and good outcomes (mRS score of 0-2). Improvement or stability of symptom and condition relative to preoperative condition was observed in 16 of 17 patients, whereas 1 patient with the petrosal type had poor outcome caused by postembolic hemorrhage. This patient underwent emergency craniotomy using the retrosigmoid approach to remove intratumoral massive hemorrhage 2 hours after embolization. No recurrence has been observed among the 16 resected hemangioblastomas at the last follow-up visit with mean follow-up_period of $38.1 \pm 22.5$ months (range, 5-72). Further surgery was required for another lesion in two patients with VHL because of tumor enlargement during the follow-uppperiod.

\section{Discussion}

\section{Classifications of Cerebellar Hemangioblastomas Based on the Venous Drainage System}

The present study proposed an anatomical classification of cerebellar hemangioblastomas based on the venous drainage system. Identification of the exact origin of the tumor is essential for optimum selection of the surgical approach for cerebellar hemangioblastomas, but determination on only_preoperative MR images may be difficult due to deformation of the cerebellar surface by the tumor, peritumoral cyst, and perifocal edema, especially with larger tumors. Our classification found a strikingly limited subset of a unique drainage system relative to the tumor origin in all hemangioblastomas. This unique drainage system consisted of peculiar large inherent veins located at the origin of the cerebellar hemangioblastoma and forming the main drainage passing into specific dural venous sinuses. Such a drainage system passing through a single large draining vein into one of the venous sinuses is similar to the patterns found in arteriovenous malformations (AVMs). [35,36]

Cerebellar hemangioblastomas arise at various sites in the cerebellum with characteristic vascular architectures. The main histopathological feature of hemangioblastomas is the presence of two main components consisting of neoplastic stromal cells and abundant vascular cells.[14] Recent studies demonstrated that these stromal cells represent the cytologic equivalent of embryonal hemangioblasts, which are common progenitor cells with differentiation potential into endothelial cells and hematopoietic 
stem cells. $[6,31,32]$ Precirculatory vascular plexuses derived from hemangioblastic cell islands eventually establish communication through the arterial and venous systems.[35] The nature of the active process remains unknown, but such an embryonal vascular plexus might connect to the regional draining veins emptying into the adjacent venous sinuses.

The classification of the cerebellar veins is based on three cortical surfaces, containing the superior hemispheric and superior vermian veins from the tentorial surface, inferior hemispheric and inferior vermian veins from the suboccipital surface, and petrosal veins from the petrosal surface. These superficial cerebellar veins terminate as bridging veins collecting into three major venous systems, the galenic, petrosal, and tentorial venous systems. The superficial veins of the galenic group include the precentral cerebellar veins, the superior vermian vein, and the anterior group of the superior hemispheric vein, that drain the anterior part of the tentorial surface. The petrosal draining group includes the superficial veins that drain the lateral part of the cerebellar hemisphere, collecting into the SPSs. The tentorial draining group includes the superficial veins draining the suboccipital surface and the posterior part of the tentorial surface, which collect into the straight and the transverse sinuses and the torcula, either directly or through a tentorial sinus. The cerebellar tonsil has two unique but different drainage systems. The superficial venous system includes the superior and inferior retrotonsillar veins which join to form the inferior vermian vein, emptying into the transverse sinuses and the torcula. The deep venous system including the supratonsillar veins drains into the vein of the cerebellomedullary fissure anastomosing with the vein of the inferior cerebellar peduncle, which directly drains into the jugular bulb or into the SPS via the vein of the middle cerebellarpeduncle.[22,23] Accordingly, we proposed the anatomical classification of cerebellar hemangioblastomas based on the venous drainage system, and indicated the appropriate surgical approach. In our series, suboccipital and tentorial hemangioblastomas were associated with the tentorial draining group from the suboccipital surface and the posterior part of the tentorial surface, respectively. Petrosal and quadrigeminal hemangioblastomas were associated with the petrosal draining group and the galenic group, respectively. Tonsillar hemangioblastoma was separately categorized from the suboccipital type because of the association with a deep venous drainage system.

Surgery for hemangioblastomas is regarded as difficult due to their AVM-like characteristics.[15,21] Venous drainage and eloquence are key components of the Spetzler-Martin grading system, but these factors are not a reliable indicator of surgical risks of cerebellar AVM because of the specific anatomical properties of the cerebellum. Tumor location and supplementary grading system are important for predicting outcomes after cerebellar AVM resection.[24] However, the most recent data from multicenter retrospective analysis indicated a significantly greater risk for poor outcomes in patients with deep venous drainage into the galenic system,[18] which is an excellent indicator of cerebral AVM depth in the Spetzler-Martin grading system.[28] Cerebellar AVMs can be classified into 5 subtypes, vermian, suboccipital, tentorial, petrosal, and tonsillar according to their location.[24] This anatomical classification might be adapted to cerebellar hemangioblastomas, because the drainage system might consist of unique large venous drainage similar to cerebellar AVMs.[35,36] 


\section{Selection of the Surgical Approach}

Safe resection of hemangioblastomas requires careful selection of the surgical approach to achieve panoramic exposure which allows early identification of the major feeding arteries and single main draining vein, and direct visualization of any small feeding arteries around the entire pial border, especially for large, solid tumor with deep location. Posterior fossa hemangioblastomas can be divided into four types according to their location and feeding arteries. Type I is found in the cerebellar hemisphere and vermis fed by the SCA, PICA, and AICA, type II in the cerebellar tonsil fed by the AICA, PICA, and meningeal branches, type III in the fourth ventricle and brain stem fed by the PICA, and type IV in the superior vermis fed by the SCA and meningeal branches. The suboccipital ipsilateral approach, modified far-lateral approach, suboccipital midline approach, and suboccipital supracerebellar approach are applied to tumor types I, II, III and IV, respectively.[5,33] In our experience, the anatomy of the arterial blood supply is not important to select the surgical approach because most large, solid hemangioblastomas receive multiple arterial blood supplies around the entire tumor-pial border or tumorneural tissue interface from the SCA, AICA, and PICA, regardless of tumor origin and location in the cerebellum. Selection of surgical approach according to venous drainage system is important because this characteristic exclusively reflects the tumor origin.

Suboccipital hemangioblastomas were most common type (ㄴ․%) in this study. Cerebellar hemangioblastomas predominantly located in posterior half of the cerebellum.[8] Suboccipital hemangioblastoma is most accessible and can be excised through standard suboccipital craniotomy matching the tumor size and location. Tentorial hemangioblastomas were second most common type $(21.1 \%)$ in this study, and could be resected through the OTA or SCITA to gain access to the entire tumorpial margin on the superior cerebellar surface. Quadrigeminal hemangioblastomas were found in $10.5 \%$ of cases in this study. This type of hemangioblastoma is the most challenging because of the deepest location in the superior vermis and anteromedial region of the superior cerebellum facing the quadrigeminal plate, accessed through the OTA.

The preferred surgical approach to the upper, anterior part of the cerebellum is still controversial. The OTA and SCITA are the most commonly used among the various reported surgical approaches[5,7-

$9,11,20,24,26,27,33]$ and the subtemporal transtentorial approach is used for some limited cases.[2,3] We performed the OTA for 3 tentorial and 2 quadrigeminal hemangioblastomas with midline location, and the SCITA for one tentorial hemangioblastomas with lateral extension. We preferentially used the OTA for tentorial and quadrigeminal hemangioblastomas, because of the excellent, panoramic exposure of the entire tumor-pial border on the superior cerebellar surface, early management of the SCA, and direct visualization of the draining veins into the straight sinus or galenic system throughout surgery. Injury of venous drainage system is important to avoid during incision of the tentorium. In this study, the cerebellar hemispheric veins drained directly into the straight sinus, and no lesions had direct drainage into the tentorial sinus, but the OTA may not be feasible in such cases. Risk of retraction injury of the occipital lobe can be reduced by using gravity dependent and/or intermittent retraction. The SCITA was applied for tumors with tentorial sinus drainage or lateral extension of more than $35 \mathrm{~mm}$ and contralateral extension 
of more than $17 \mathrm{~mm}$ from the midline, which is the limit of the OTA.[11] Petrosal hemangioblastoma was found in $10.5 \%$ of cases in this study, but may also include the rarely reported so-called cerebellopontine angle hemangioblastoma, so little information is available about the details of the venous drainage system. We treated petrosal hemangioblastomas through retrosigmoid craniotomies with exposure of the transverse-sigmoid junction to gain access to the petrosal surface and SPS, as previously reported. $[1,15,21,24]$ Tonsillar hemangioblastomas were found in $10.5 \%$ of cases in this study. Midline suboccipital craniotomy including the posterior lip of the foramen magnum as well as the posterior arch of $\mathrm{C} 1$ could provide wide exposure of the dissection plane and allow access to the deep drainage system at the bottom of the tumor adjacent to the lateral recess of the fourth ventricle.

\section{Clinical Outcome}

This study showed that improvement or stable symptoms and condition relative to preoperative findings were observed in $94.1 \%$ of patients and hydrocephalus was resolved in all cases. A previous study demonstrated that stable or improved conditions were found immediately after $88 \%$ of tumor resections and hydrocephalus resolved in $94 \%$ of patients after selective tumor resection.[8] One patient in our series had poor outcome, who experienced intratumoral hemorrhage immediately after the preoperative liquid embolization with NBCA. This procedure is useful and important for reduction of arterial blood supply and intraoperative bleeding,[16,17,29,30] but formidable, severe embolic complications have been reported due to the unique properties of the posterior fossa.[25] Surgery after preoperative liquid embolization on the same day is safe and effective for solid cerebellar hemangioblastoma.[12] The timing of surgery and optimal indication should be considered to prevent any complication due to tumor swelling, intratumoral hemorrhage, or adjacent cerebellar infarction after preoperative embolization. No major complications occurred related to the surgical procedures and no single approach had a significantly higher incidence of postoperative neurological deficits. Therefore, no patient had experienced recurrence of the resected lesions at the last follow-up visit. Our classification based on the venous drainage system might be beneficial and helpful to select the optimal surgical approach for cerebellar hemangioblastomas, especially larger tumor with extensive perifocal edema.

\section{Limitation}

Potential limitations of this study include the relatively small number of patients who were not randomly assigned prospectively to each study group.

\section{Conclusions}

Surgical removal of cerebellar hemangioblastomas remains extremely challenging due to their characteristics. Careful consideration of the optimal surgical approach is quite important for en-bloc gross total resection with sparing of the surrounding neurovascular structures. Division of the cerebellar surface into five different regions based on the venous drainage system will help the surgeon to select the ideal surgical approach and guide surgical decision making for cerebellar hemangioblastomas. 


\section{Declarations}

\section{Funding}

Not applicable

\section{Conflict of interest}

The authors report no conflict of interest concerning the materials or methods used in this study or the findings specified in this paper.

\section{Availability of data and material}

Not applicable

\section{Code availability}

Not applicable

\section{Ethical approval}

All procedures performed in studies involving human participants were in accordance with the ethical standards of the institutional and/or national research committee and with the 1964 Helsinki declaration and its later amendments or comparable ethical standards.

This study was approved by the regional ethics committee of the Faculty of Medicine, University of Miyazaki (No. 2020-225).

\section{Consent to participate}

Informed consent was provided before intervention by each patient and his/her nearest family member.

\section{Consent for publication}

Patient consent was also obtained for publication of images or other clinical information of the patient in a medical publication, journal, website, medical book, or other forms of publication with maximum efforts to conceal his/her identity. All authors of this manuscript also consent to the publication of the manuscript in Neurosurgical Review journal.

\section{Author Contributions}

Conception and design: Watanabe. Acquisition of data: Watanabe, Suematsu, Yamashita. Analysis and interpretation of data: Watanabe, Ohta, Yokogami, Takeshima. Drafting the article: Watanabe. Critically revising the article: all authors. Reviewed submitted version of manuscript: Watanabe. Statistical 
analysis: Watanabe. Material support: Suematsu, Saito, Takeishi. Study supervision: Watanabe, Takeshima.

\section{References}

1. Amano T, Tokunaga S, Shono T, Mizoguchi M, Matsumoto K, Yoshida F, Sasaki T (2009) Cerebellar hemangioblastoma manifesting as hearing disturbance. Neurol Med Chir (Tokyo) 49:418-420. https://doi.org/10.2176/nmc.49.418

2. Ammerman JM, Lonser RR, Oldfield EH (2005) Posterior subtemporal transtentorial approach to intraparenchymal lesions of the anteromedial region of the superior cerebellum. J Neurosurg 103:783-788. https://doi.org/10.3171/jns.2005.103.5.0783

3. Beppu T, Hirooka R, Fujiwara S, Kashimura H, Nishimoto H, Ogasawara K, Ogawa A (2009) Choice of posterior subtemporal transtentorial approach for tumor resection in deep anteromedial superior cerebellum. Neurol Med Chir (Tokyo) 49:42-46. https://doi.org/10.2176/nmc.49.42

4. Cavenee WK, Louis DN, Ohgaki H, Wiestler OD (2007) WHO Classification of Tumours of the Central Nervous System, 4th edition. Lyon, IARC.

5. Cui H, Zou J, Bao YH, Wang MS, Wang Y (2017) Surgical treatment of solid hemangioblastomas of the posterior fossa: A report of 28 cases. Oncol Lett 13:1125-1130. https://doi.org/10.3892/ol.2016.5531

6. Glasker S, Li J, Xia JB, Okamoto H, Zeng W, Lonser RR, Zhuang Z, Oldfield EH, Vortmeyer AO (2006) Hemangioblastomas share protein expression with embryonal hemangioblast progenitor cell. Cancer Res 66:4167-4172. https://doi.org/10.1158/0008-5472.can-05-3505

7. Isolan GR, KrayenbühI N, Mahmoud M, Al-Mefty O (2007) A hemangioblastoma in the pineal region: case report. Neurosurgery 61:E423; discussion E423. https://doi.org/10.1227/01.neu.0000255516.12085.bb

8. Jagannathan J, Lonser RR, Smith R, DeVroom HL, Oldfield EH (2008) Surgical management of cerebellar hemangioblastomas in patients with von Hippel-Lindau disease. J Neurosurg 108:210222. https://doi.org/10.3171/jns/2008/108/2/0210

9. Kawauchi D, Higuchi Y, Ikegami S, Adachi A, Ozaki K, Suda I, Kobayashi E, Iwadate Y (2019) Unilateral Occipital Transtentorial Approach with Multimodal Assistance for Resection of Large Supracerebellar Hemangioblastomas: Preliminary Experience of 2 Cases. World Neurosurg 129:e733e740. https://doi.org/10.1016/j.wneu.2019.06.001

10. Kuharic M, Jankovic D, Splavski B, Boop FA, Arnautovic KI (2018) Hemangioblastomas of the Posterior Cranial Fossa in Adults: Demographics, Clinical, Morphologic, Pathologic, Surgical Features, and Outcomes. A Systematic Review. World Neurosurg 110:e1049-e1062. https://doi.org/10.1016/j.wneu.2017.11.173

11. Kurokawa Y, Uede T, Hashi K (1999) Operative approach to mediosuperior cerebellar tumors: occipital interhemispheric transtentorial approach. Surg Neurol 51:421-425. https://doi.org/10.1016/s0090- 
3019(98)00123-2

12. Kuwahara K, Ichikawa T, Haruma J, Hishikawa T, Hiramatsu M, Sugiu K, Date I (2017) [Preoperative Embolization for Solid Cerebellar Hemangioblastoma on the Day of Surgery:Two Case Reports]. No Shinkei Geka 45:615-622. https://doi.org/10.11477/mf.1436203560

13. Lonser RR, Glenn GM, Walther M, Chew EY, Libutti SK, Linehan WM, Oldfield EH (2003) von HippelLindau disease. Lancet 361:2059-2067. https://doi.org/10.1016/s0140-6736(03)13643-4

14. Louis DN OH, Wiestler OD, Cavenee WK (2016) WHO Classification of Tumours of the Central Nervous System, Revised 4th Edition. Lyon, IARC.

15. Moon BH, Park SK, Han YM (2014) Large solid hemangioblastoma in the cerebellopontine angle: complete resection using the transcondylar fossa approach. Brain Tumor Res Treat 2:128-131. https://doi.org/10.14791/btrt.2014.2.2.128

16. Munyon C, Chowdhry SA, Cohen ML, Bambakidis NC, Hsu DP (2011) N-butyl 2-cyanoacrylate (n-BCA) embolization of a cerebellar hemangioblastoma. J Neurointerv Surg 3:386-389 https://doi.org/10.1136/jnis.2010.004366

17. Murai Y, Kominami S, Yoshida Y, Mizunari T, Adachi K, Koketsu K, Kobayashi S, Teramoto A (2012) Preoperative liquid embolization of cerebeller hemangioblastomas using $\mathrm{N}$-butyl cyanoacrylate. Neuroradiology 54:981-988. https://doi.org/10.1007/s00234-011-0985-5

18. Nisson PL, Fard SA, Walter CM, Johnstone CM, Mooney MA, Tayebi Meybodi A, Lang M, Kim H, Jahnke H, Roe DJ, Dumont TM, Lemole GM, Spetzler RF, Lawton MT (2019) A novel proposed grading system for cerebellar arteriovenous malformations. J Neurosurg:1-11. https://doi.org/10.3171/2018.12.jns181677

19. Niu L, Zhang Y, Li Q, Dai J, Yin H, Duan L, Yang H, Liang W, Qin Z, Zhang J, Pan Y (2016) The analysis of correlative factors affecting long-term outcomes in patients with Solid Cerebellar Hemangioblastomas. Clin Neurol Neurosurg 150:59-66.

https://doi.org/10.1016/j.clineuro.2016.08.028

20. Rabadán AT, Hernández DA, Paz L (2016) Hemangioblastoma located in the posterior incisural space mimicking a tentorial meningioma: a case report. J Med Case Rep 10:183.

https://doi.org/10.1186/s13256-016-0962-z

21. Rachinger J, Buslei R, Prell J, Strauss C (2009) Solid haemangioblastomas of the CNS: a review of 17 consecutive cases. Neurosurg Rev 32:37-47; discussion 47-38. https://doi.org/10.1007/s10143008-0166-0

22. Rhoton AL, Jr. (2000) Cerebellum and fourth ventricle. Neurosurgery 47:S7-27 https://doi.org/10.1097/00006123-200009001-00007

23. Rhoton AL, Jr. (2000) The posterior fossa veins. Neurosurgery 47:S69-92 https://doi.org/10.1097/00006123-200009001-00012

24. Rodriguez-Hernandez A, Kim H, Pourmohamad T, Young WL, Lawton MT (2012) Cerebellar arteriovenous malformations: anatomic subtypes, surgical results, and increased predictive accuracy 
of the supplementary grading system. Neurosurgery 71:1111-1124.

https://doi.org/10.1227/NEU.0b013e318271c081

25. Sakamoto N, Ishikawa E, Nakai Y, Akutsu H, Yamamoto T, Nakai K, Shiigai M, Tsurushima H, Isobe T, Takano S, Tsuboi K, Matsumura A (2012) Preoperative endovascular embolization for hemangioblastoma in the posterior fossa. Neurol Med Chir (Tokyo) 52:878-884. https://doi.org/10.2176/nmc.52.878

26. Sanai N, Mirzadeh Z, Lawton MT (2010) Supracerebellar-supratrochlear and infratentorialinfratrochlear approaches: gravity-dependent variations of the lateral approach over the cerebellum. Neurosurgery 66:264-274; discussion 274. https://doi.org/10.1227/01.neu.0000369653.12185.fd

27. Shirane R, Kumabe T, Yoshida Y, Su CC, Jokura H, Umezawa K, Yoshimoto T (2001) Surgical treatment of posterior fossa tumors via the occipital transtentorial approach: evaluation of operative safety and results in 14 patients with anterosuperior cerebellar tumors. J Neurosurg 94:927-935. https://doi.org/10.3171/jns.2001.94.6.0927

28. Spetzler RF, Martin NA (1986) A proposed grading system for arteriovenous malformations. J Neurosurg 65:476-483. https://doi.org/10.3171/jns.1986.65.4.0476

29. Sultan A, Hassan T, Aboul-Enein H, Mansour O, Ibrahim T (2016) The value of preoperative embolization in large and giant solid cerebellar hemangioblastomas. Interv Neuroradiol 22:482-488 https://doi.org/10.1177/1591019916633244

30. Takeuchi S, Tanaka R, Fujii Y, Abe H, Ito Y (2001) Surgical treatment of hemangioblastomas with presurgical endovascular embolization. Neurol Med Chir (Tokyo) 41:246-251; discussion 251-242. https://doi.org/10.2176/nmc.41.246

31. Vortmeyer AO, Frank S, Jeong SY, Yuan K, Ikejiri B, Lee YS, Bhowmick D, Lonser RR, Smith R, Rodgers G, Oldfield EH, Zhuang Z (2003) Developmental arrest of angioblastic lineage initiates tumorigenesis in von Hippel-Lindau disease. Cancer Res 63:7051-7055.

32. Vortmeyer AO, Yuan Q, Lee YS, Zhuang Z, Oldfield EH (2004) Developmental effects of von HippelLindau gene deficiency. Ann Neurol 55:721-728. https://doi.org/10.1002/ana.20090

33. Wan JQ, Cui H, Wang Y (2011) Surgical management of large solid hemangioblastomas of the posterior fossa. J Clin Neurosci 18:39-42. https://doi.org/10.1016/j.jocn.2010.07.099

34. Wanebo JE, Lonser RR, Glenn GM, Oldfield EH (2003) The natural history of hemangioblastomas of the central nervous system in patients with von Hippel-Lindau disease. J Neurosurg 98:82-94. https://doi.org/10.3171/jns.2003.98.1.0082

35. Yasargil M (1987) Microneurosurgery, volume IIIA; AVM of the Brain, History, Embryology, Pathological Considerations, Hemodynamics, Diagnostic Studies, Microsurgical Anatomy. Stuttgart, Thieme.

36. Yasargil M (1988) Microneurosurgery, IIIB; AVM of the brain, Clinical Considerations, General and Special Operative Techniques, Surgical Results, Nonoperated Cases, Cavernous and Venous Angiomas, Neuroanesthesia. Stuttgart, Thieme. 


\section{Tables}

Table 1. Demographics of 17 patients with cerebellar hemangioblastomas

\begin{tabular}{|ll|}
\hline Characteristic & Value \\
\hline Age, mean \pm SD (range), yrs & $53.6 \pm 20.8$ (23-86) \\
\hline Sex & \\
\hline Male & 10 \\
\hline Female & 7 \\
\hline Diagnosis & \\
\hline VHL & 9 \\
\hline Sporadic & 8 \\
\hline Initial symptom & \\
\hline Cerebellar ataxia & 10 \\
\hline Headache & 5 \\
\hline Facial numbness & 1 \\
\hline Disturbance of consciousness & 1 \\
\hline Asymptomatic & 3 \\
\hline
\end{tabular}

SD = standard deviation.

Table 2. Characteristics of 19 cerebellar hemangioblastomas 


\begin{tabular}{|ll|}
\hline Characteristics & Value \\
\hline Morphologic types, $\mathrm{n}(\%)$ & \\
\hline Solid & $9(47.4)$ \\
\hline Peritumoral cyst & $8(42.1)$ \\
\hline Intratumoral cyst & $2(10.5)$ \\
\hline Size & \\
\hline Maximum diameter, mean \pm SD, mm & $33.7 \pm 12.9$ \\
\hline Large $\geq 25$ mm, $\mathrm{n}(\%)$ & $15(78.9)$ \\
\hline Small <25 mm, $\mathrm{n}(\%)$ & $4(21.1)$ \\
\hline Lesion, $\mathrm{n}(\%)$ & \\
\hline Solitary & $11(57.9)$ \\
\hline Multiple & $8(42.1)$ \\
\hline Hydrocephalus, $\mathrm{n}(\%)$ & \\
\hline Yes & $13(68.4)$ \\
\hline No & $6(31.6)$ \\
\hline Peritumoral edema, $\mathrm{n}(\%)$ & $38.1 \pm 22.5$ \\
\hline Extensive & $11(63.2)$ \\
\hline Local & \\
\hline No & \\
\hline Preoperative embolization, $\mathrm{n}(\%)$ & \\
\hline Yes & \\
\hline No & \\
\hline Mean follow-up, mean $\pm \mathrm{SD}, \mathrm{month})$ \\
\hline
\end{tabular}

Table 3. Characteristics of five subtypes of cerebellar hemangioblastomas 


\begin{tabular}{|c|c|c|c|c|c|}
\hline & Suboccipital & Tentorial & Petrosal & Quadrigeminal & Tonsillar \\
\hline $\begin{array}{l}\text { No. of lesions, } n \\
\text { (\%) }\end{array}$ & $9(47.4)$ & $4(21.1)$ & $2(10.5)$ & $2(10.5)$ & $2(10.5)$ \\
\hline \multicolumn{6}{|l|}{$\begin{array}{l}\text { Morphology, n } \\
\text { (\%) }\end{array}$} \\
\hline Solid & $3(36.4)$ & $3(75)$ & $1(50)$ & $1(50)$ & $1(50)$ \\
\hline Peritumoral cyst & $5(54.5)$ & $1(25)$ & $1(50)$ & 0 & $1(50)$ \\
\hline $\begin{array}{l}\text { Intratumoral } \\
\text { cyst }\end{array}$ & $1(9)$ & 0 & 0 & $1(50)$ & 0 \\
\hline $\begin{array}{l}\text { Mean maximum } \\
\text { diameter, } \mathrm{mm}\end{array}$ & 33.1 & 32 & 38 & 39.5 & 30.5 \\
\hline \multirow[t]{2}{*}{$\begin{array}{l}\text { Surgical } \\
\text { approach }\end{array}$} & \multirow[t]{2}{*}{$\begin{array}{l}\text { Standard } \\
\text { suboccipital }\end{array}$} & OTA $(n=3)$ & \multirow[t]{2}{*}{ Retrosigmoid } & \multirow[t]{2}{*}{ OTA } & \multirow{2}{*}{$\begin{array}{l}\text { Midline } \\
\text { suboccipital } \\
\text { C-1 } \\
\text { laminectomy }\end{array}$} \\
\hline & & SCITA $(n=1)$ & & & \\
\hline Draining veins & IVV, IHV & SHV & PV & SVV, PrCV & RTV, STV \\
\hline Venous system & TS, Torc & SS & SPS & GS & $\begin{array}{l}\text { Torc, } \\
\text { JB/SPS }\end{array}$ \\
\hline \multicolumn{6}{|l|}{$\begin{array}{l}\text { Extent of } \\
\text { resection, n (\%) }\end{array}$} \\
\hline Gross total & 8 (98.9) & $4(100)$ & $2(100)$ & $2(100)$ & $2(100)$ \\
\hline Subtotal & $1(11.1)$ & 0 & 0 & 0 & 0 \\
\hline \multicolumn{6}{|l|}{$\begin{array}{l}\text { Surgical } \\
\text { complication }\end{array}$} \\
\hline Transient & None & $\begin{array}{l}\text { Hemianopsia } \\
(\mathrm{n}=3)\end{array}$ & None & $\begin{array}{l}\text { Trochlear } \\
\text { nerve palsy } \\
(n=1)\end{array}$ & None \\
\hline Persistent & None & None & None & $\begin{array}{l}\text { Hemianopsia } \\
(\mathrm{n}=1)\end{array}$ & None \\
\hline $\begin{array}{l}\text { IVR-related } \\
\text { complication }\end{array}$ & None & None & $\mathrm{IH}(\mathrm{n}=1)$ & None & None \\
\hline \multicolumn{6}{|l|}{$\begin{array}{l}\text { Clinical } \\
\text { outcome, n (\%) }\end{array}$} \\
\hline $\begin{array}{l}\text { Improved or } \\
\text { same }\end{array}$ & $9(100)$ & $4(100)$ & $1(50)$ & $2(100)$ & $2(100)$ \\
\hline Worsened & 0 & 0 & $1(50)$ & 0 & 0 \\
\hline
\end{tabular}


$\mathrm{GS}$ = galenic system; IVV = inferior vermian venin; IHV = inferior hemispheric vein; JB= Jugular bulb, PV = petrosal vein; SHV = superior hemispheric vein; $\mathrm{SPS}$ = superior petrosal sinus; $\mathrm{SS}$ = straight sinus; $\mathrm{TS}$ = tentorial sinus; SVV = superior vermian vein; IVR = interventional radiology; IH = intratumoral hemorrhage; $\mathrm{RTV}=$ retrotonsillar vein; STV=Supratonsillar vein

\section{Figures}
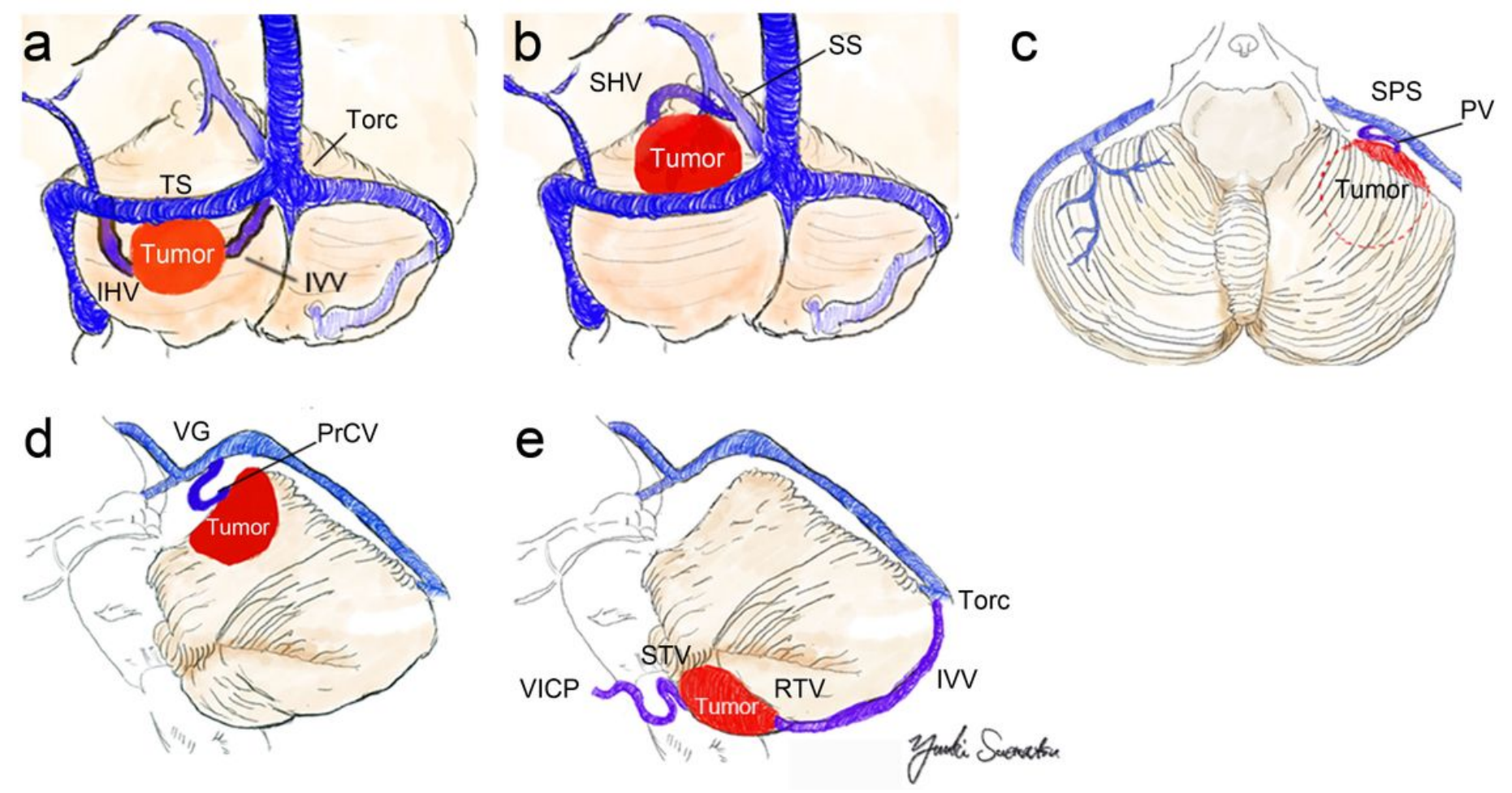

\section{Figure 1}

Artist's illustration depicting the cerebellar hemangioblastoma classification based on the venous drainage system. Suboccipital hemangioblastoma (a) is located on the suboccipital surface and drained by the inferior vermian veins (IVV), inferior hemispheric veins (IHV), or both, which empty into the transverse sinus (TS) or torcular (Torc). Tentorial hemangioblastoma (b) faces the tentorial surface along the supracerebellar-infratentorial plane, and is drained through the superior hemispheric veins (SHV) passing into the straight sinus (SS). Petrosal hemangioblastoma (c) faces the corner of the petrosal surface and tentorium, and drained by the petrosal vein (PV) emptying into the superior petrosal sinus (SPS). Quadrigeminal hemangioblastoma (d) is located on the anteromedial cerebellar surface facing the quadrigeminal cistern, and drained by the superior vermian vein or precentral cerebellar vein (PrCV) passing into the vein of Galen (VG). Tonsillar hemangioblastoma (e) is located on the tonsillar surface and drained through the retrotonsillar vein (RTV) joining to the IVV, emptying into torcular, and through the supratonsillar vein (STV) anastomosing with the vein of the inferior cerebellar peduncle (VICP) into the jugular bulb (JB) or SPS. 

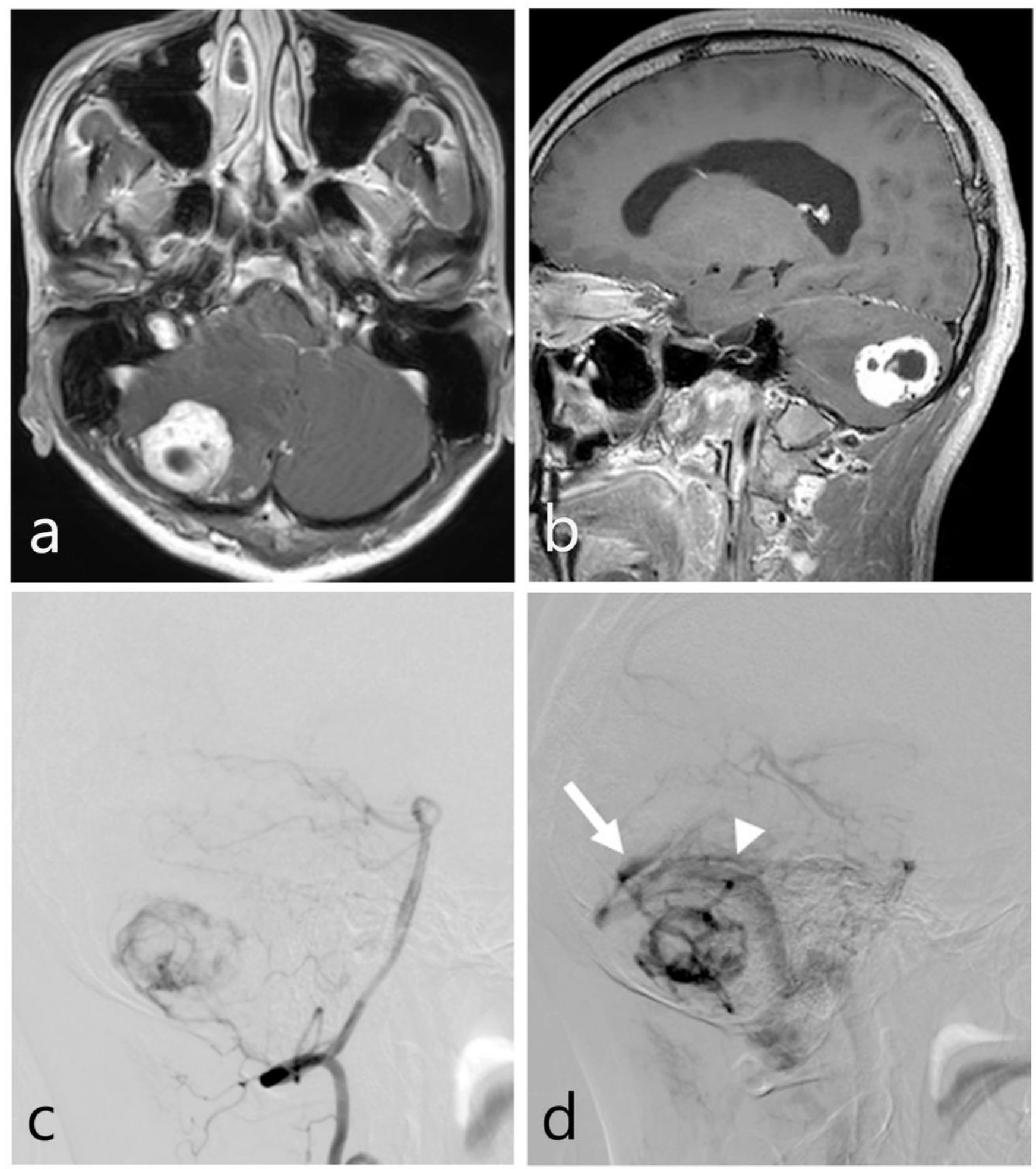

\section{Figure 2}

Axial (a) and sagittal (b) T1-weighted gadolinium-enhanced magnetic resonance images showing a large suboccipital hemangioblastoma. Digital subtraction angiograms $(c, d)$ demonstrating drainage into the torcular and transverse sinus through the inferior vermian vein and inferior hemispheric vein (arrow) (arrowhead), respectively. 

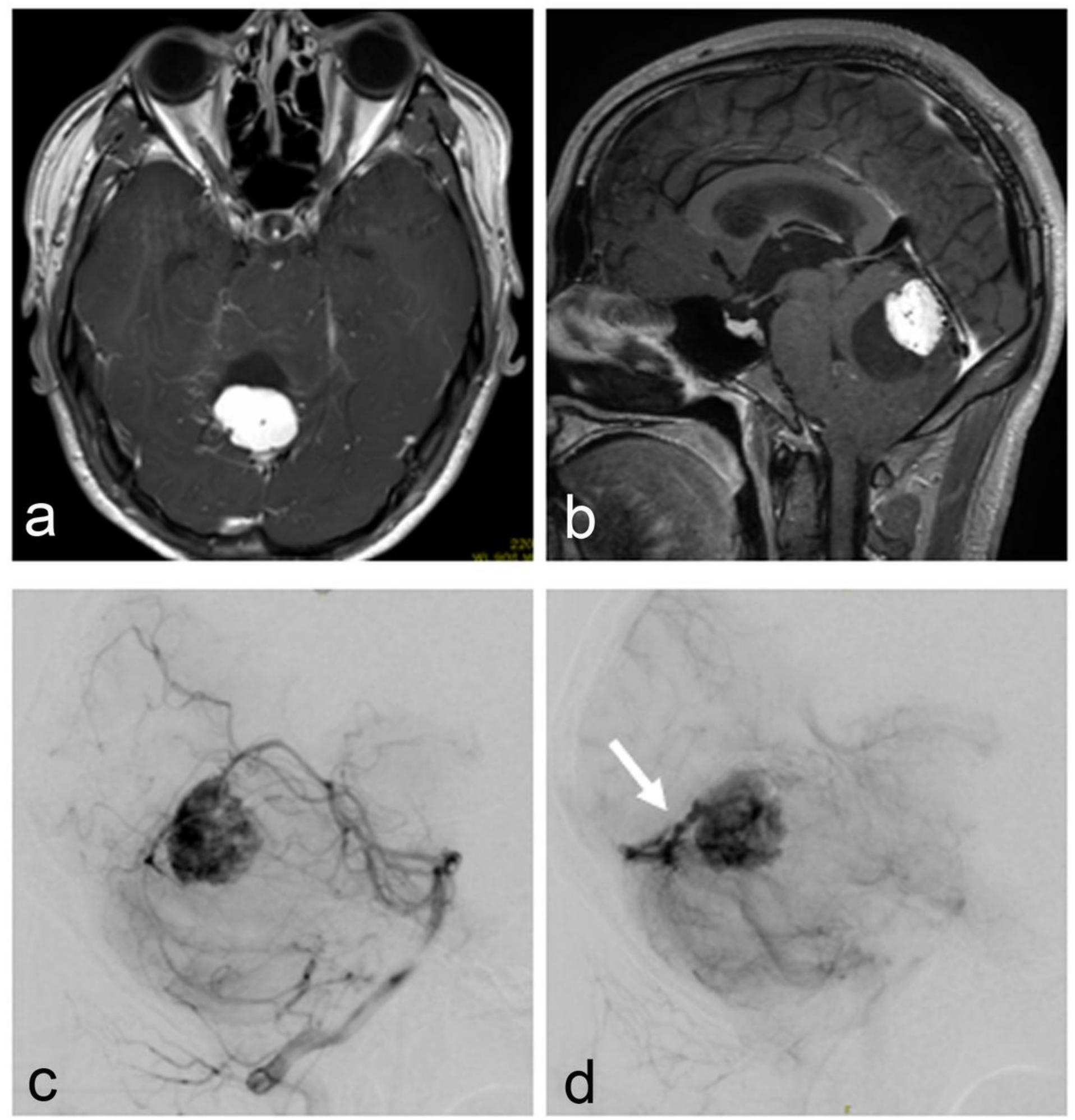

\section{Figure 3}

Axial (a) and sagittal (b) T1-weighted gadolinium-enhanced magnetic resonance images showing a tentorial hemangioblastoma. Digital subtraction angiograms $(c, d)$ demonstrating drainage into the superior hemispheric vein (arrow) emptying into the straight sinus. 

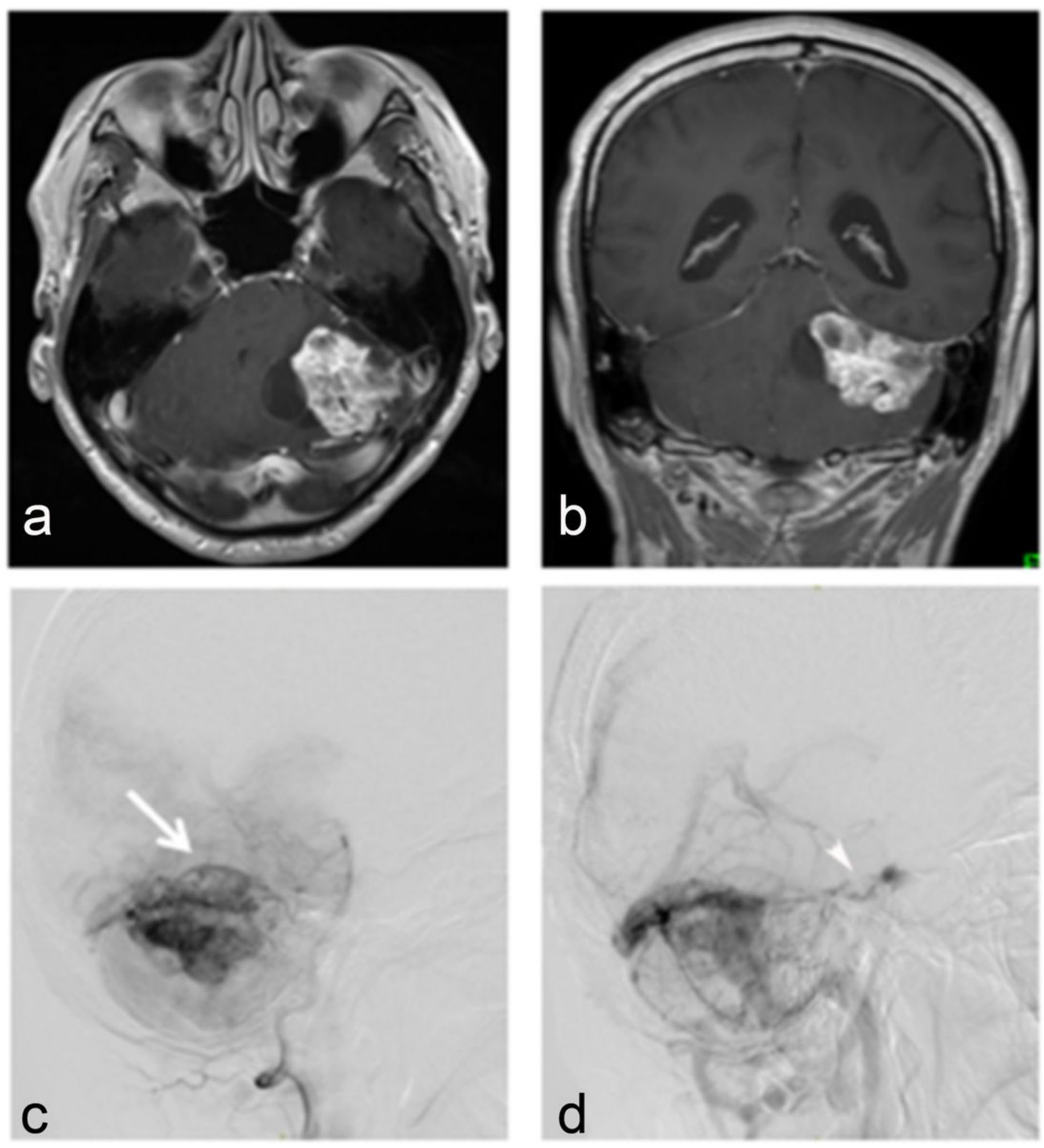

\section{Figure 4}

Axial (a) and coronal (b) T1-weighted gadolinium-enhanced magnetic resonance images showing a petrosal hemangioblastoma. Digital subtraction angiograms $(c, d)$ demonstrating drainage into the superior sagittal sinus through the petrosal vein (arrow). 

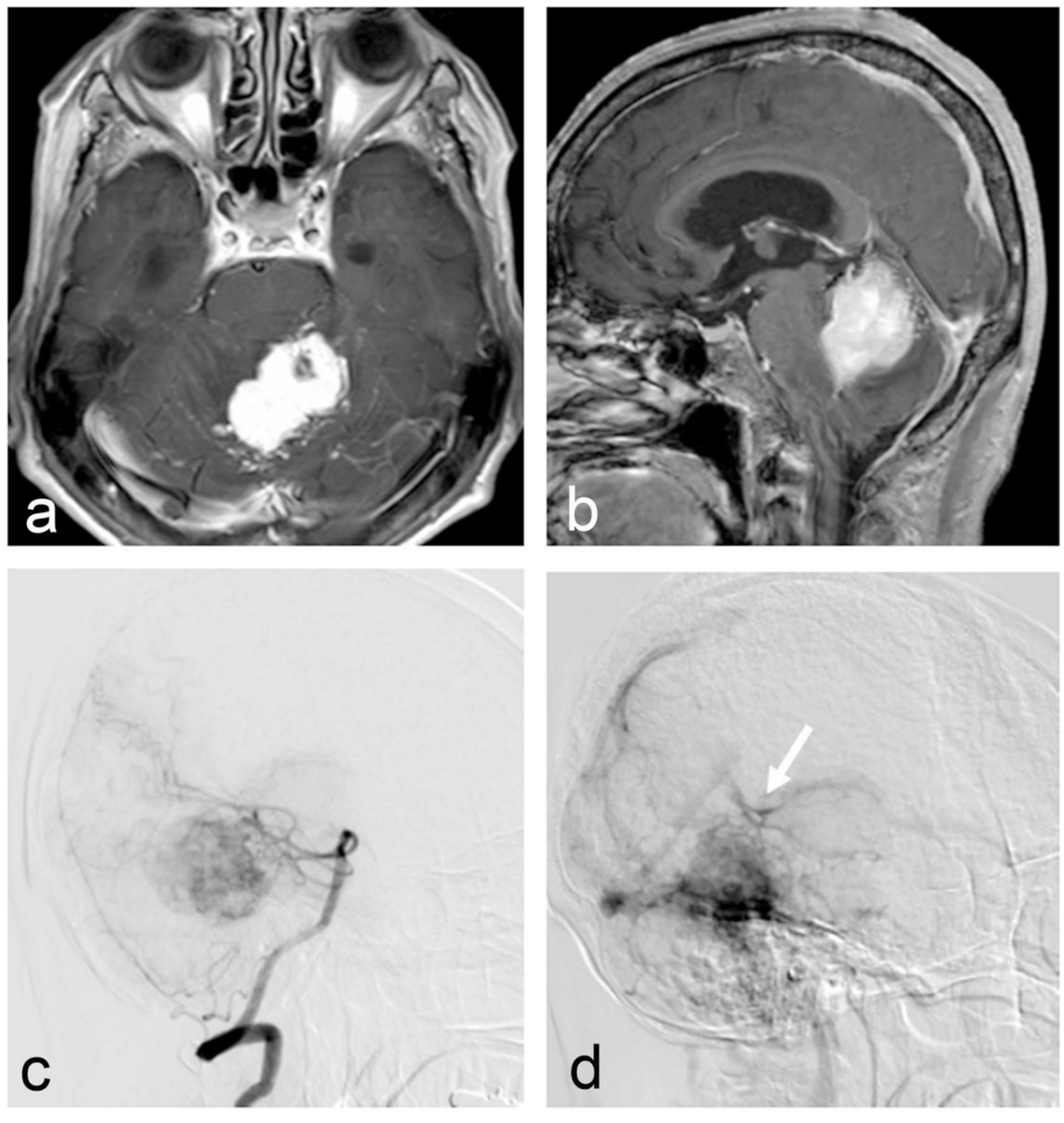

\section{Figure 5}

Axial (a) and sagittal (b) T1-weighted gadolinium-enhanced magnetic resonance images showing a quadrigeminal hemangioblastoma with intratumoral cyst. Digital subtraction angiograms (c, d) demonstrating drainage into the precentral cerebellar vein (arrow) emptying into the vein of Galen. 

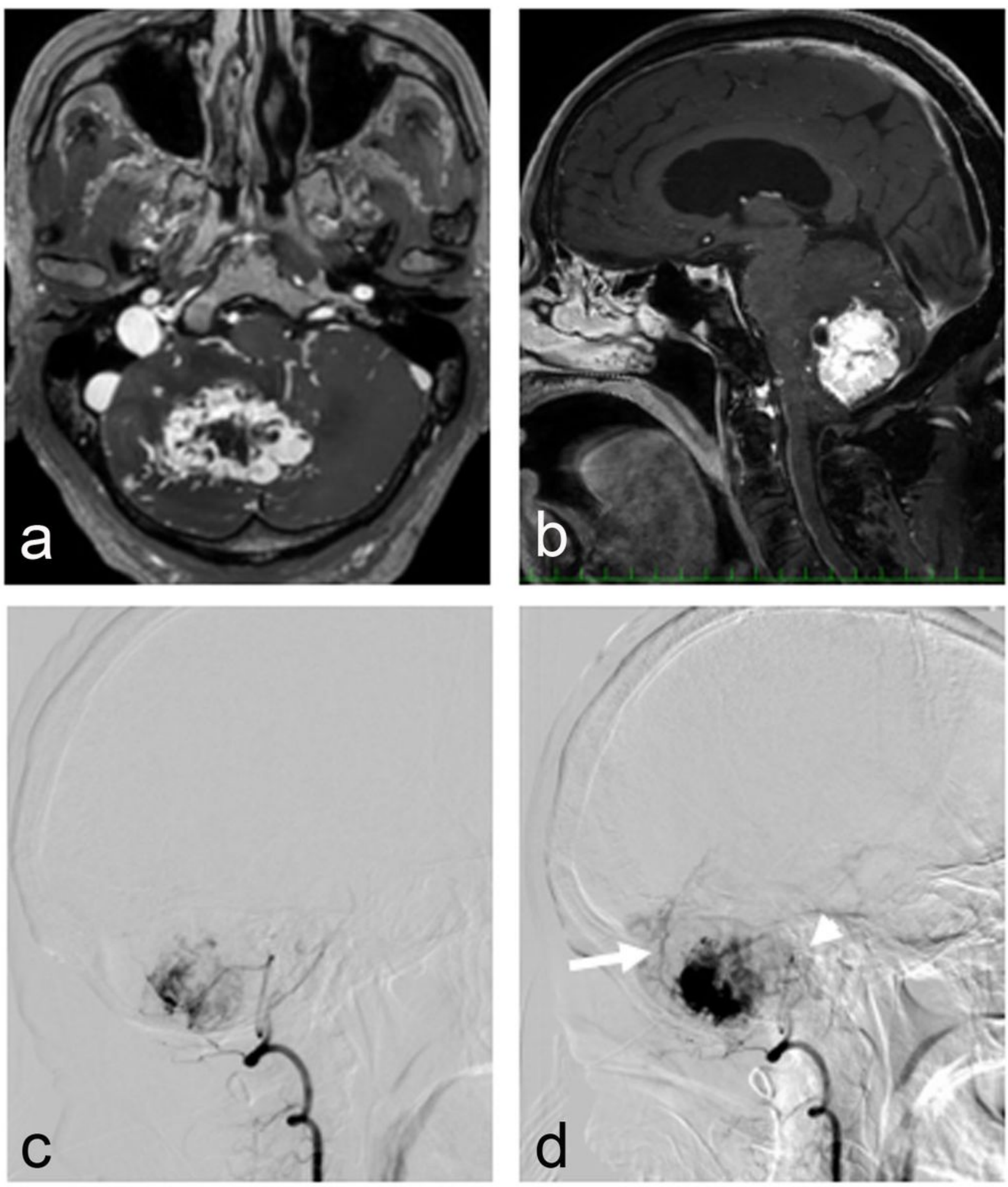

\section{Figure 6}

Axial (a) and sagittal (b) T1-weighted gadolinium-enhanced magnetic resonance images showing a large tonsillar hemangioblastoma. Digital subtraction angiograms $(c, d)$ demonstrating drainage into the torcular and jugular bulb through the inferior vermian vein (arrow) and vein of the inferior cerebellar peduncle (arrowhead), respectively. 